\title{
Laser calibration of the ATLAS Tile Calorimeter
}

\author{
Giulia Di Gregorio*† \\ INFN-Università di Pisa \\ E-mail: giulia.di.gregorio@cern.ch
}

High performance stability of the ATLAS Tile calorimeter is achieved with a set of calibration procedures. One step of the calibration procedure is based on measurements of the response stability to laser excitation of the photomultipliers (PMTs) that are used to readout the calorimeter cells. A facility to study in lab the PMT stability response is operating in the PISA-INFN laboratories since 2015. Goals of the tests in lab are to study the time evolution of the PMT response to reproduce and to understand the origin of the response drifts seen with the PMT mounted on the Tile calorimeter in its normal operation during LHC Run 1 and Run 2.

A new statistical approach was developed to measure the drift of the absolute gain. This approach was applied to both the ATLAS laser calibration data and to the data collected in the Pisa local laboratory. The preliminary results from these two studies are discussed.

The European Physical Society Conference on High Energy Physics

5-12 July, 2017

Venice

* Speaker.

${ }^{\dagger}$ On behalf of the ATLAS Tile calorimeter System 


\section{Introduction}

The ATLAS Tile Calorimeter (TileCal) [1] is the barrel section of the hadronic calorimeter of the ATLAS experiments and provides important information for reconstruction of hadrons, jets, hadronic decays of $\tau$ leptons and missing transverse energy. It is a sampling calorimeter made up of scintillating tiles as active material and steel plates as absorbers. The light produced by the passage of particles is transmitted to readout PMTs by wavelength shifting fibres. The analog signals are used for trigger purposes. The digitized signals are processed with an algorithm in the ReadOut Drivers (RODs) and propagated to the ATLAS ReadOut Systems.

\section{Calibration systems and time stability of the PMT response at ATLAS}

To maintain the calorimeter high performance, it is required a continuous percent-level calibration of each cell. The TileCal response is calibrated via three systems (Figure 1) [2]:

1. Cesium radioactive source system to equalize the response of all cells and to calibrate the global response of the calorimeter at the electromagnetic scale.

2. Laser calibration system to measure the stability of the PMTs response between two subsequent Cesium calibrations.

3. Charge Injection system to calibrate the response of the ADCs.

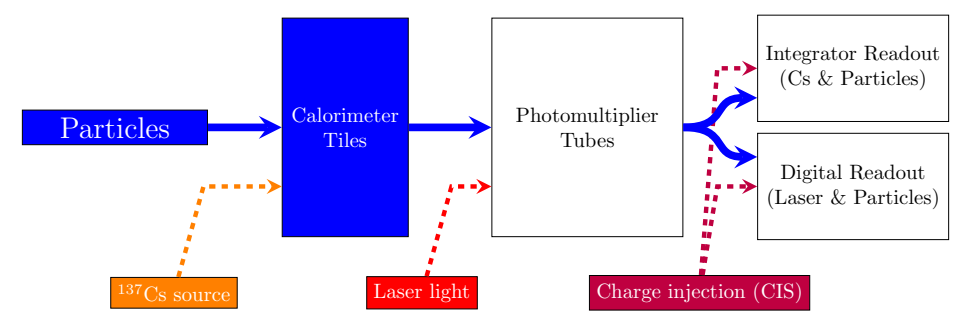

Figure 1: Scheme of the TileCal calibration chain [3].

The three calibration systems monitor independently the different parts of the readout chain. Additionally, Minimum Bias (MB) events, "Particles" in Figure 1, are used to provide an independent cross-check of Cs global calibration.

The Laser system monitors both the PMTs and the front-end electronics used for data acquisition. Light pulses, similar to those produced by ionizing particles, are transmitted simultaneously at all the TileCal PMTs through an optical system including a bundle of 100 meter long clear fibres. The Laser system is used to monitor the PMT response between two Cs scans.

\section{Time stability of the PMT response at ATLAS}

Figures 2(a) and 2(b) show the average variation of the A13 and E4 cells response to laser pulses (blue points) and to MB events (green points). The observation period ranges from May $24^{\text {th }} 2016$ (day of last detector calibration with Cesium source system ${ }^{1}$, indicated with a vertical

\footnotetext{
${ }^{1}$ In 2016 the Cs scans were suspended due to a water leakage.
} 
red dashed line in the figure) to October $27^{\text {th }} 2016$ (end of $p-p$ collisions). The variations observed in the MB events depends on PMT drift and scintillator irradiation. The difference between MB events and Laser pulses is interpreted as the effect of the scintillators irradiation.

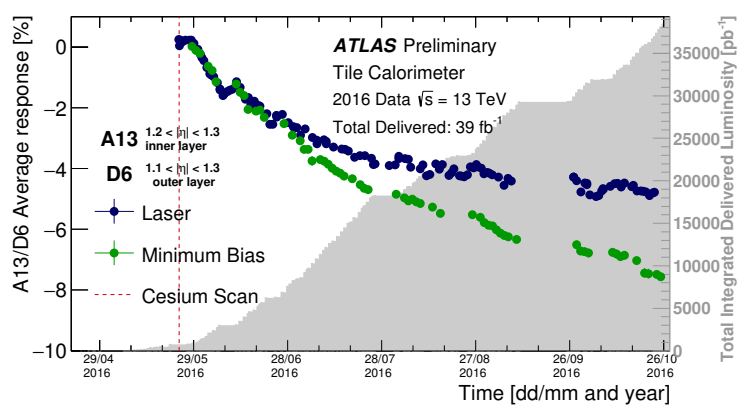

(a)

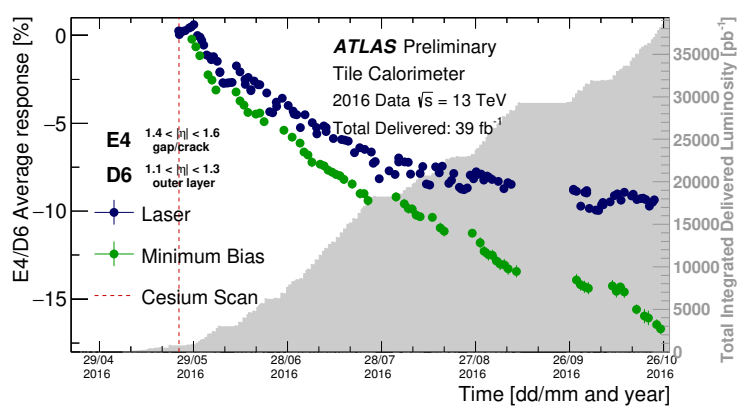

(b)

Figure 2: Evolution of A13/D6 (a) and E4/D6 (b) cell response to laser pulses (blue points) and Minimum Bias events (green points). The integrated luminosity is shown with grey color on the background. The response variation has been normalized to the PMT response measured on the last day of Cs calibration [3].

Figure 3 shows the average down-drift of each cell type in the observation period. The average PMT response variation is defined as the mean of the Gaussian function that fits the PMT variation distribution of all channels associated to the same calorimeter cell type. The observed down-drift mostly affects cells in the inner radius that are the cells with the higher exposure. The maximum down-drift is of the order of $-14 \%$. Differently the cells in the outer radius are mostly stable. These results are obtained applying the official laser calibration procedure as described in Ref. [2].

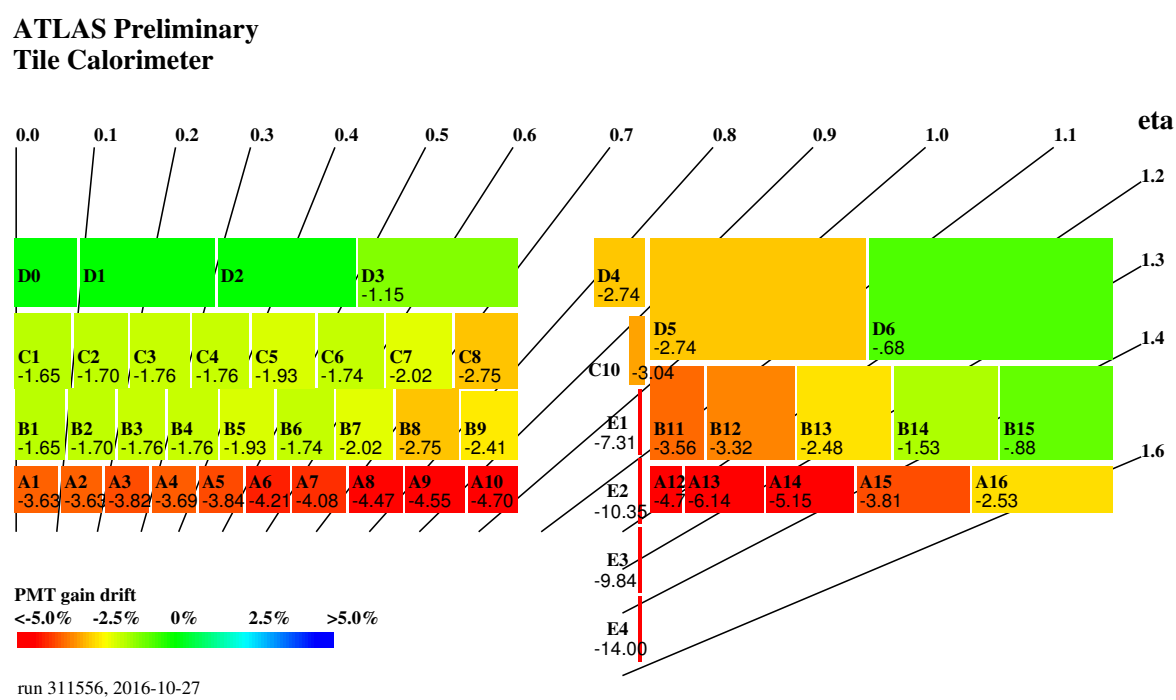

Figure 3: TileCal cell map with the PMT drift values in the considered period [3]. 


\section{The statistical method for PMT gain measurements}

A method, based on the statistical model of photoelectron production and multiplication inside a PMT, is used to measure the PMT gain. Since the electronic noise can be neglected, the two main contributions to the PMT signal fluctuations are the photoelectron emission and multiplication, described by a Poisson statistics, and the intensity variation of the light source [4]. So this give:

$$
\frac{\operatorname{Var}(q)}{\langle q\rangle^{2}}=f \cdot \frac{\sigma_{N}^{2}}{\langle N\rangle^{2}}+\frac{\operatorname{Var}(I)}{\langle I\rangle^{2}}
$$

where $\langle q\rangle(\operatorname{Var}(q))$ is the average (variance) anode charge, $\langle N\rangle\left(\sigma_{N}^{2}\right)$ is the average (variance) number of photoelectrons, $\langle I\rangle(\operatorname{Var}(I))$ is the average (variance) light intensity of a pulsed source on the cathode; $f$ is the excess noise factor. The excess noise factor $f$ is a measure of the deviation of the observed PMT resolution from the expected based on Poisson photo-statistics. The average anode charge $\langle q\rangle$ and the average of photoelectrons $\langle N\rangle$ are related by $\langle q\rangle=e \cdot G \cdot\langle N\rangle$. One has:

$$
G=\frac{1}{f \cdot e}\left(\frac{\operatorname{Var}(q)}{\langle q\rangle}-k\langle q\rangle\right)
$$

where $k=\frac{\operatorname{Var}(I)}{\langle I\rangle^{2}}$ is the coherence factor which depends only on the light properties and it is unknown. To estimate this value, one can use the formula 4.2 at two different light intensity corresponding to charge values $q_{1}$ and $q_{2}$ (intensity method):

$$
k=\frac{\frac{\operatorname{Var}\left(q_{1}\right)}{\left\langle q_{1}\right\rangle}-\frac{\operatorname{Var}\left(q_{2}\right)}{\left\langle q_{2}\right\rangle}}{\left\langle q_{1}\right\rangle-\left\langle q_{2}\right\rangle}
$$

An alternative method to evaluate $k$ relies on the fact that several PMTs are used to measure the light from the same source ( covariance method). Since by definition $k=\frac{\operatorname{Var}(I)}{\langle I\rangle^{2}}$, for any PMT pair identified by indices $i$ and $j$, the $k$ parameter can be expressed as:

$$
k=\frac{\operatorname{Cov}\left(q_{i}, q_{j}\right)}{\left\langle q_{i}\right\rangle\left\langle q_{j}\right\rangle}
$$

It is evaluated from the mean signal of each PMT pair receiving similar fractions of the same light pulses at fixed intensity.

\subsection{The stability of PMTs response and gain}

Figure 4 shows the evolution in lab measurements of the PMT gain of four never used PMTs model Hamamatsu R11187, an evolution of R7877 mounted on TileCal. The factor $k$ used to evaluate the gain is calculated with the intensity method (open circles) and with the covariance method (full circles) [5] discussed in the previous section. On day 20/01/2017 the PMT HV was increased from $700 \mathrm{~V}$ to $830 \mathrm{~V}$. The expected increase of the gain by a factor of about 1.5-2.5 is measured in all the cases. The errors comes for the linear fit of the energy scan (4 points) and from the distribution of the gain measured from the covariance of 7 independent pairs (14 PMTs). The covariance method appears to be more precise and a general agreement between the two methods is observed at both voltages. Nine PMTs, dismounted from TileCal detector in February 2017 


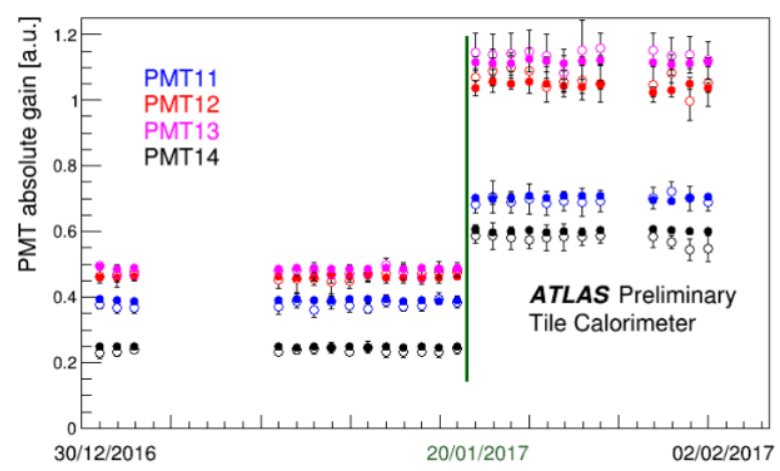

Figure 4: PMT absolute gain at different HV values. Open circles shows the gain calculated using intensity scan method, full circle shows the gain calculated using the covariance method [3].

(model Hamamatsu R7877) are studied in the Pisa-INFN laboratory. These PMTs were reading out the signals of different cell types (A, BC, D, E). They have an integrated anode charge ranging from 1 to $5 \mathrm{C}$ during Run 1 and Run 2. In the Pisa set-up anode charge integration is achieved using a pulsed green LED, while PMT response is measured with a pulsed laser ( 80 ps pulse width, $\lambda=432 \mathrm{~nm}$ ). Figure 5 shows the time evolution of the average PMT response (black points) and average PMT absolute gain (red points) normalized to the first day of observation and to the signal of a reference PMT monitoring the light source intensity. Error bars include statistical and systematic (dominant) contributions. The two time evolutions overlap within the error bars; this fact may indicate that the drift of the PMT response is due to the drift of the PMT absolute gain. However, since the PMT absolute gain drift is systematically smaller than the PMT response drift, the difference between the two curves could be ascribed to other effects such as cathode quantum efficiency loss and/or PMT windows transparency degradation.

In the test bench operation the average integrated charge is $20 \mathrm{C}$ and the typical down-drift per integrated charge is about $-0.2 \% / \mathrm{C}$ at the end of the observation period. The average down-drift of the PMT response per unit of integrated charge measured at the test bench is compatible with the corresponding down-drift observed for the response of the PMTs installed on the calorimeter.

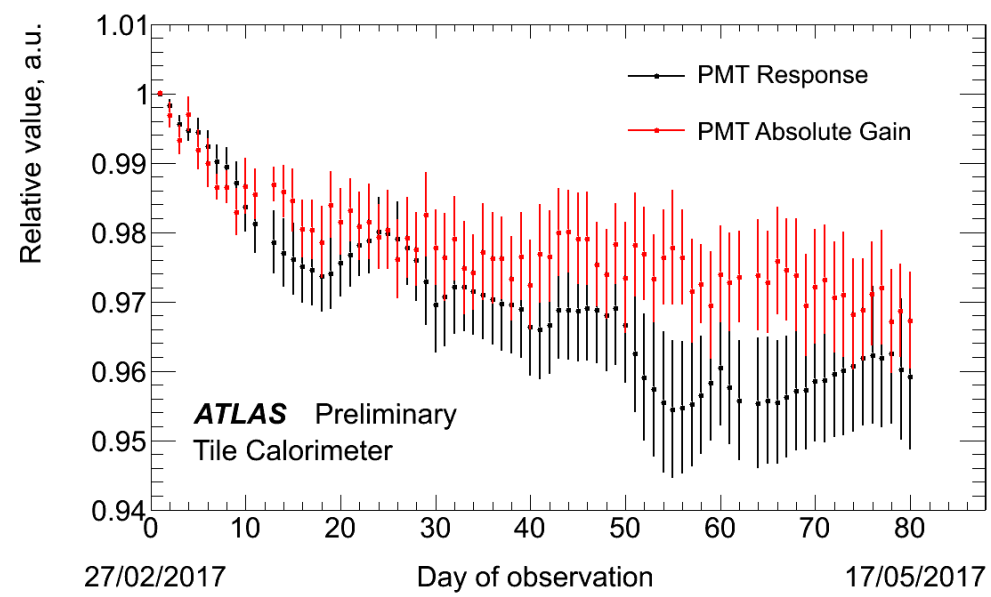

Figure 5: Time evolution of the PMT response (black) and PMT absolute gain (red) [3]. 


\section{Conclusions}

In 2016 data taking period the PMT response obtained using the Laser system is characterized by a down-drift which depends on cell exposure. The maximum down-drift is of the order of $-14 \%$ for the cells in the inner radius. A facility to study the PMT stability is operating in PisaINFN laboratory. To understand the origin of the response drifts seen with the PMT mounted on TileCal, a new statistical approach is used to measure the PMT absolute gain. Preliminary results confirm that the down-drift is mainly due to the gain variation.

\section{References}

[1] ATLAS Collaboration, The ATLAS Experiment at the CERN Large Hadron Collider, JINST 3 (2008) S08003.

[2] D. Boumediene et al., Calibration of the Tile Hadronic Calorimeter of ATLAS at LHC, J. Phys. Conf. Ser. 587 (2015) 1, 012009.

[3] ATLAS Tile Calorimeter Subsystem, Approved plots. https://twiki.cern.ch/twiki/bin/view/AtlasPublic/ApprovedPlotsTile

[4] J. Bures, Degeneracy of light and the optimum accurancy of photoelectric measurements, Journal of the Optical Society of America, Vol. 64, N. 12 (1974).

[5] V.F. Kazanin, Laboratory test of the response stability of the ATLAS Tile Calorimeter photomultipliers, ATL-TILECAL-PROC-2017-009. 